\title{
Differential Hopf algebra structures on the universal enveloping algebra of a Lie algebra
}

\author{
N. van den Hijligenberg ${ }^{a)}$ and R. Martinib) \\ CWI. P.O. Box 94079, 1090 GB Amsterdam, The Netherlands
}

(Received 5 July 1995; accepted for publication 25 July 1995)

\begin{abstract}
We discuss a method to construct a De Rham complex (differential algebra) of Poincaré-Birkhoff-Witt type on the universal enveloping algebra of a Lie algebra $\mathfrak{g}$. We determine the cases in which this gives rise to a differential Hopf algebra that naturally extends the Hopf algebra structure of $U(\mathfrak{g})$. The construction of such differential structures is interpreted in terms of color Lie superalgebras. () 1996 American Institute of Physics. [S0022-2488(95)02112-5]
\end{abstract}

\section{INTRODUCTION}

Recently noncommutative differential geometry has attracted considerable interest, both mathematically and as a framework for certain models in theoretical physics. In particular, there is much activity in differential geometry on quantum groups. A noncommutative differential calculus on quantum groups has been developed by Woronowicz ${ }^{1}$ following general ideas of Connes. ${ }^{2}$ This general theory has been reformulated by Wess and Zumino ${ }^{3}$ in a less abstract way. Their approach may be more suitable for specific applications in physics. A large number of papers have been written since and a few other methods to construct a noncommutative differential geometry on a quantum group or to define a differential geometric structure (a De Rham complex) on a given noncommutative algebra have been proposed and discussed by several authors (e.g., Refs. 4-6).

In this paper we present a differential calculus on the enveloping algebra of a given Lie algebra. This differential structure turns out to be a differential Hopf algebra, which can be interpreted in a very interesting way in terms of color Lie superalgebras. The commutative case has been studied previously (see, e.g., Ref. 7). Notice that our approach is different from the standard methods to construct noncommutative differential structures on Hopf algebras and quantum groups, in the sense that our starting point is not the algebra of functions on the (quantum) group but its dual the (quantized) universal enveloping algebra.

In the classical limit a quantized universal enveloping algebra defines a co-Poisson-Hopf algebra structure on the universal enveloping algebra (see Ref. 8). Further research is in progress concerning the compatibility between the differential calculus and the Poisson cobracket in order to define a differential Hopf algebra structure on the quantized universal enveloping algebra. We will report on this in the near future.

\section{A DE RHAM COMPLEX ON $U(\mathfrak{g})$}

Let $A$ be an associative algebra over the field of complex numbers. A differential algebra on $A$ (or a De Rham complex on $A$, see Ref. 4 ) is an $\mathbb{N}$-graded associative algebra $\Omega$ equipped with a linear operator $d$ that has the following properties: (1) $d$ is homogeneous of degree 1 , i.e. $d\left(\Omega^{p}\right) \subset \Omega^{p+1}$ for all $p \in \mathbb{N}$. (2) $d$ is a differential, i.e. $d^{2}=d \circ d=0$. (3) $d$ is a graded derivation (of degree 1 ), i.e.

$$
d(a b)=d(a) b+(-1)^{p} a d(b), \quad a \in \Omega^{p}, \quad b \in \Omega .
$$

\footnotetext{
a) Supported by NWO Grant No. 611-307-100.

b) Present address: University of Twente, Department of Applied Mathematics, The Netherlands.
} 
Furthermore, the algebra $\Omega$ has to be generated by $\Omega^{0} \cup d\left(\Omega^{0}\right)$, where $\Omega^{0}$ is isomorphic to $A$. For $A$ we take the universal enveloping algebra of a Lie algebra and discuss the construction of De Rham complexes on such an algebra.

Let $g$ be a finite-dimensional Lie algebra over $\mathrm{C}$ with basis $\left\{x^{1}, x^{2}, \ldots, x^{n}\right\}$ and corresponding structure constants $C_{k}^{i j}$, which are defined by the property $\left[x^{i}, x^{j}\right]=C_{k}^{i j} x^{k}$. Throughout this paper we will make use of the Einstein summation convention. The universal enveloping algebra of $\mathfrak{g}$, which we denote by $U(\mathfrak{g})$, can be viewed as the quotient algebra of the free associative algebra on the alphabet $\left\{x^{1}, x^{2}, \ldots, x^{n}\right\}$ modulo the ideal generated by the relations

$$
x^{i} x^{j}-x^{j} x^{i}=C_{k}^{i j} x^{k}
$$

From the Poincaré-Birkhoff-Witt Theorem we know that the monomials $x^{i_{1}} x^{i_{2}} \cdots x^{i_{p}}$ with $p \geqslant 0$ and $i_{1} \leqslant i_{2} \leqslant \cdots \leqslant i_{p}$ form a basis of $U(\mathfrak{g})$. The main idea behind the construction of $\Omega$ on $U(\mathfrak{g})$ is that we demand $\Omega$ to be of the PBW type; by this we mean that the monomials

$$
d x^{j_{1}} d x^{j_{2}} \cdots d x^{j_{q}} x^{i_{1}} x^{i_{2}} \cdots x^{i_{p}} \text {, with } j_{1}<j_{2}<\cdots<j_{q}, \quad i_{1} \leqslant i_{2} \leqslant \cdots \leqslant i_{p}, \quad p, q \geqslant 0,
$$

are a basis of the associative algebra $\Omega$. From here on we will write $y^{j}$ to denote the element $d x^{j}=d\left(x^{j}\right)$ in $\Omega^{1}$.

In order to construct $\Omega$ we have to impose certain commutation relations between the elements $x^{i}$ and $y^{j}$. On account of the homogeneity and the PBW property, we impose relations of the form

$$
x^{i} y^{j}=y^{k}\left(\Theta_{l k}^{i j} x^{l}+A_{k}^{i j}\right)
$$

where both $\Theta_{l k}^{i j}$ and $A_{k}^{i j}$ are arbitrary complex numbers. Since we want to obtain an ideal that is invariant under the action of the differential, we adjoin the following commutation relations for the elements $y^{i}$ and $y^{j}$ :

$$
y^{i} y^{j}=-\Theta_{l k}^{i j} y^{k} y^{l}
$$

This is simply the consequence of applying $d$ to the relations (4). Let $\Omega$ be the quotient algebra of the free associative algebra on the alphabet $\left\{x^{1}, x^{2}, \ldots, x^{n}, y^{1}, y^{2}, \ldots, y^{n}\right\}$ modulo the ideal $J$ that is generated by the relations (2), (4), and (5). The $N$ grading of $\Omega$ is induced by giving the elements $x^{i}$ degree 0 and the elements $y^{j}$ degree 1 . The differential $d$ is defined by $d\left(x^{i}\right)=y^{i}, d\left(y^{i}\right)=0$ and the derivation property (1).

The coefficients $\Theta_{l k}^{i j}$ and $A_{k}^{i j}$ should satisfy a number of conditions in order that $\Omega$ has the above mentioned properties of a De Rham complex on $U(\mathfrak{g})$. The first condition arises from $d(J) \subset J$, i.e. the $d$ invariance of the ideal $J$. By applying $d$ to (2) we obtain

$$
y^{i} x^{j}+x^{i} y^{j}-y^{j} x^{i}-x^{j} y^{i}=C_{k}^{i j} y^{k}
$$

which, on account of the relations (4), can be written as

$$
y^{i} x^{j}+y^{k}\left(\Theta_{l k}^{i j} x^{l}+A_{k}^{i j}\right)-y^{j} x^{i}-y^{k}\left(\Theta_{l k}^{j i} x^{l}+A_{k}^{j i}\right)=C_{k}^{i j} y^{k} .
$$

The PBW property of $\Omega$ implies that

$$
A_{k}^{i j}-A_{k}^{j i}=C_{k}^{i j} \quad \text { and } \quad y^{i} x^{j}-y^{j} x^{i}+\left(\Theta_{l k}^{i j}-\Theta_{l k}^{j i}\right) y^{k} x^{l}=0 .
$$

We can write this in the following compact form:

$$
(I-P) A=C \quad \text { and } \quad I-P+\Theta P-P \Theta P=(I-P)(I+\Theta P)=0 .
$$


and its multiplication $\mu_{\otimes}: \Omega \otimes \Omega \otimes \Omega \otimes \Omega \rightarrow \Omega \otimes \Omega$ by

$$
\mu_{\otimes}=(\mu \otimes \mu) \circ \sigma_{23},
$$

where $\mu$ denotes the multiplication of $\Omega$. The linear map $\sigma_{23}$ denotes the graded flip $\sigma: \Omega \otimes \Omega \rightarrow \Omega \otimes \Omega$, which is defined by

$$
\sigma(a \otimes b)=(-1)^{p q} b \otimes a, \quad a \in \Omega^{p}, \quad b \in \Omega^{q},
$$

applied to the second and third component of the tensor product. The differential $d_{\otimes}$ of $\Omega \otimes \Omega$ is given by $d_{\otimes}=d \otimes i d+\tau \otimes d$, where $\tau: \Omega \rightarrow \Omega$ is the linear map of degree zero satisfying $\tau(a)=(-1)^{p} a$ for all $a \in \Omega^{p}$. Written out explicitly, this gives

$$
d_{\otimes}(a \otimes b)=d(a) \otimes b+(-1)^{p} a \otimes d(b), \quad a \in \Omega^{p}, \quad b \in \Omega .
$$

Henceforth, the condition that $\Delta$ should be a differential algebra morphism means that $\Delta$ is an algebra morphism with the property

$$
d_{\otimes} \circ \Delta=(d \otimes i d+\tau \otimes d) \circ \Delta=\Delta \circ d .
$$

Note that this is the analog of the derivation property (1) that can be written as $d \circ \mu=\mu^{\circ} d_{\otimes}$. We consider $\mathbb{C}$ to be a differential algebra with $\mathbb{C}^{0}=\mathbb{C}$ and $\mathbb{C}^{p}=0$ for all $p>0$. Hence, $\epsilon$ is a differential algebra morphism if and only if it is an algebra morphism satisfying $\epsilon^{\circ} d=0$.

Since $\Omega$ is generated by $\Omega^{0} \cup d\left(\Omega^{0}\right)$, it suffices to define the actions of $\Delta$ and $\epsilon$ on $\left\{x^{1}, x^{2}, \ldots, x^{n}, y^{1}, y^{2}, \ldots, y^{n}\right\}$. Naturally the actions on elements $x^{i}$ coincide with the previously described actions on $U(\mathfrak{g})$. The above mentioned conditions for $\Delta$ and $\epsilon$ uniquely determine the actions on the elements $y^{j}$ :

$$
\begin{aligned}
\Delta\left(y^{i}\right)=\Delta \circ d\left(x^{i}\right)=d_{\otimes} \Delta\left(x^{i}\right) & =d_{\otimes}\left(x^{i} \otimes 1+1 \otimes x^{i}\right)=y^{i} \otimes 1+1 \otimes y^{i}, \\
\epsilon\left(y^{i}\right) & =\epsilon \circ d\left(x^{i}\right)=0 .
\end{aligned}
$$

Here we have used that $d(1)=0$, which is a direct consequence of the derivation property of $d$.

In order for $\epsilon$ and $\Delta$ to be well defined, the ideal $J$ needs to be a (two-sided) coideal, i.e. $\epsilon(J)=0$ and $\Delta(J) \subset \Omega \otimes J+J \otimes \Omega$. The first condition is clearly satisfied. To verify the second we apply $\Delta$ to the relations (2), (4), and (5). Naturally the relations (2) do not give rise to any conditions for the coefficients $\Theta_{l k}^{i j}$ and $A_{k}^{i j}$. The relations (4) yield

$$
\begin{aligned}
\Delta\left(x^{i} y^{j}-\Theta_{l k}^{i j} y^{k}\left(x^{l}+A_{k}^{i j}\right)\right)= & \Delta\left(x^{i}\right) \Delta\left(y^{j}\right)-\Theta_{l k}^{i j} \Delta\left(y^{k}\right)\left(\Delta\left(x^{l}\right)+A_{k}^{i j} 1 \otimes 1\right) \\
= & 1 \otimes x^{i} y^{j}+x^{i} \otimes y^{j}+y^{j} \otimes x^{i}+x^{i} y^{j} \otimes 1 \\
& -\Theta_{l k}^{i j}\left(1 \otimes y^{k} x^{l}+x^{l} \otimes y^{k}+y^{k} \otimes x^{l}+y^{k} x^{l} \otimes 1+A_{k}^{i j}\left(1 \otimes y^{k}+y^{k} \otimes 1\right)\right) \\
= & x^{i} \otimes y^{j}+y^{j} \otimes x^{i}-\Theta_{l k}^{i j}\left(y^{k} \otimes x^{l}+x^{l} \otimes y^{k}\right) \bmod (\Omega \otimes J+J \otimes \Omega) .
\end{aligned}
$$

Again, on account of the PBW property of $\Omega$, we obtain

$$
x^{i} \otimes y^{j}=\Theta_{l k}^{i j} x^{l} \otimes y^{k} \quad \text { and } \quad y^{j} \otimes x^{i}=\Theta_{l k}^{i j} y^{k} \otimes x^{l},
$$

which evidently implies $\Theta=I$. Similarly, we find

$$
\Delta\left(y^{i} y^{j}+\Theta_{l k}^{i j} y^{k} y^{l}\right)=y^{i} \otimes y^{j}-y^{j} \otimes y^{i}+\Theta_{l k}^{i j}\left(y^{k} \otimes y^{l}-y^{l} \otimes y^{k}\right) \bmod (J \otimes \Omega+\Omega \otimes J),
$$


which boils down to the condition $(I-\Theta)(I-P)=0$. Therefore, the conclusion is that $\Omega$ can only be a differential bialgebra on $U(\mathfrak{g})$ if $\Theta=I$. In that case the conditions (6), (7), (8), and (9) reduce to

$$
(I-P) A=C \quad \text { and }\left[A^{j}, A^{i}\right]=C_{l}^{i j} A^{l},
$$

due to the fact that $P^{2}=I$ and $S^{2}=I$. The commutation relations (4) and (5) take the following form:

$$
x^{i} y^{j}=y^{j} x^{i}+A_{k}^{i j} y^{k} \quad \text { and } \quad y^{i} y^{j}=-y^{j} y^{i}
$$

We remark that we did not check the coassociativity of $\Delta$, i.e. $(\Delta \otimes i d) \circ \Delta=($ id $\otimes \Delta) \circ \Delta$. This is a direct consequence of the coassociativity of $\Delta$ on $U(\mathfrak{g})=\Omega^{0}$ since

$$
\begin{aligned}
(\Delta \otimes i d) \circ \Delta \circ d\left(\Omega^{0}\right) & =(\Delta \otimes i d) \circ d_{\otimes} \circ\left(\Omega^{0}\right) \\
& =\left(d_{\otimes} \otimes i d+\tau \otimes \tau \otimes d\right) \circ(\Delta \otimes i d) \circ \Delta\left(\Omega^{0}\right) \\
& =\left(d \otimes i d \otimes i d+\tau \otimes d_{\otimes}\right) \circ(i d \otimes \Delta) \circ \Delta\left(\Omega^{0}\right) \\
& =(i d \otimes \Delta) \circ d_{\otimes} \circ\left(\Omega^{0}\right)=(i d \otimes \Delta) \circ \Delta \circ d\left(\Omega^{0}\right) .
\end{aligned}
$$

By a similar reasoning one can show that the counit property of $\epsilon$ on $\Omega$ is induced by the same property of $\epsilon$ on $U(\mathfrak{g})$.

The next step is to consider a differential Hopf algebra structure on $\Omega$. We recall that $\Omega$ is a differential Hopf algebra (see Ref. 4) if it is a differential bialgebra that possesses an antipode $S$. An antipode $S$ is an element of $E n d(\Omega)$ satisfying $S \star i d=i d \star S=\eta \varphi \epsilon$, where $\star$ denotes the convolution product on $E n d(\Omega)$ (see Ref. 10) defined by

$$
f \star g=\mu \circ(f \otimes g) \circ \Delta, \quad f, g \in \operatorname{End}(\Omega) .
$$

By $\eta: C \rightarrow \Omega$ we denote the unit element of $\Omega$, i.e. $\eta(1)=1$. Since $\eta \rho \epsilon$ is the unit element in $\operatorname{End}(\Omega)$ with respect to the convolution product, one can describe the antipode as the unique inverse of the identity. An important property of the antipode is that it is an antialgebra morphism; this means that $S \circ \mu^{\circ} \sigma=(S \otimes S) \circ \mu$ and $S \circ \eta=\eta$ [or equivalently $S(1)=1$ ].

We try to extend the antipode of $U(\mathfrak{g})=\Omega^{0}$ to $\Omega$. In order to do that we make the following observation. Suppose that $f$ and $g$ are homogeneous endomorphisms on $\Omega$ of degree zero that commute with the differential operator $d$. Then the properties $d_{\otimes}^{\circ} \Delta=\Delta \circ d$ and $\mu^{\circ} d_{\otimes}=d \circ \mu$ imply that

$$
\begin{aligned}
d \circ(f \star g) & =d \circ \mu \circ(f \otimes g) \circ \Delta \\
& =\mu \circ d_{\otimes} \circ(f \otimes g) \circ \Delta \\
& =\mu \circ(d \otimes i d+\tau \otimes d) \circ(f \otimes g) \circ \Delta \\
& =\mu \circ(f \otimes g) \circ(d \otimes i d+\tau \otimes d) \circ \Delta \\
& =\mu \circ(f \otimes g) \circ d_{\otimes} \circ \\
& =\mu \circ(f \otimes g) \circ \Delta \circ d=(f \star g) \circ d .
\end{aligned}
$$

Hence, the convolution product $f \star g$ also commutes with $d$. By inductive use of this argument we can conclude that $d$ commutes with $f^{n}=f \star f \star \cdots \star f$ for all positive values of $n$ if $f$ is a homoge- 
neous endomorphism of degree zero satisfying $d \circ f=f \circ d$. For $f$ we can choose the identity. This makes it plausible that $d$ commutes with $i d^{-1}=S$. So, we extend the antipode by demanding $d \circ S=S \circ d$. In particular, this yields

$$
S\left(y^{i}\right)=S \circ d\left(x^{i}\right)=d \circ S\left(x^{i}\right)=-d\left(x^{i}\right)=-y^{i} .
$$

For $S$ to be a well-defined antialgebra morphism on $\Omega$, it must leave the ideal $J$ invariant $[S(J) \subset J]$. To verify this condition, it suffices to apply $S$ to the relations $(21)$. We will only write out the first; the second can be handled similarly,

$$
\begin{aligned}
S\left(x^{i} y^{j}-y^{j} x^{i}-A_{k}^{i j} y^{k}\right) & =S\left(y^{j}\right) S\left(x^{i}\right)-S\left(x^{i}\right) S\left(y^{j}\right)-A_{k}^{i j} S\left(y^{k}\right) \\
& =y^{j} x^{i}-x^{i} y^{j}+A_{k}^{i j} y^{k} \\
& =-\left(x^{i} y^{j}-y^{j} x^{i}-A_{k}^{i j} y^{k}\right) \in J .
\end{aligned}
$$

In order to check that $S$ is indeed an antipode, we can confine ourselves to verifying the defining property of $S$ for a set of generators of $\Omega$. Since $S$ is the extension of the antipode of $U(\mathfrak{g})$, we only need to compute

$$
(S \star i d)\left(y^{i}\right)=\mu^{\circ}(S \otimes i d)\left(1 \otimes y^{i}+y^{i} \otimes 1\right)=1 \cdot y^{i}-y^{i} \cdot 1=0=\eta^{\circ} \epsilon\left(y^{i}\right) .
$$

So, at the end of this section we come to the following conclusion. In order to obtain a differential Hopf algebra $\Omega$ on $U(\mathfrak{g})$, it is necessary that the matrix $\Theta$ in (4) and (5) equals the identity matrix $I$. In that case necessary and sufficient conditions for the coefficients $A_{k}^{i j}$ are given by (20), and the corresponding Hopf algebraic extension is described by (17), (18), and (23).

\section{A LIE ALGEBRAIC INTERPRETATION}

The comultiplication of the universal enveloping algebra of a Lie algebra is cocommutative. We can easily verify that the cocommutativity of $\Delta$ on $\Omega^{0}$ gives rise to graded cocommutativity for the extension of $\Delta$ to $\Omega$ :

$$
\sigma^{\circ} \Delta \circ d\left(\Omega^{0}\right)=\sigma^{\circ} d_{\otimes} \Delta\left(\Omega^{0}\right)=d_{\otimes} \sigma^{\circ} \Delta\left(\Omega^{0}\right)=d_{\otimes}{ }^{\circ}\left(\Omega^{0}\right)=\Delta \circ d\left(\Omega^{0}\right),
$$

or, more explicitly,

$$
\sigma \circ \Delta\left(y^{i}\right)=\sigma\left(1 \otimes y^{i}+y^{i} \otimes 1\right)=y^{i} \otimes 1+1 \otimes y^{i}=\Delta\left(y^{i}\right) .
$$

We call the property $\sigma^{\circ} \Delta=\Delta$ graded cocommutativity because $\sigma$ denotes the graded flip.

It is well known (see, e.g., Ref. 11) that a cocommutative Hopf algebra $H$, which has a compatible filtering, is isomorphic to the Hopf algebra corresponding to the universal enveloping algebra of the Lie algebra of primitive elements of $H$. Note that the set of primitive elements of $H$ is defined by

$$
P(H)=\{x \in H \mid \Delta(x)=1 \otimes x+x \otimes 1\},
$$

which has the structure of a Lie algebra with the commutator $[x, y]=x y-y x$. By a compatible filtering we mean an increasing family of subspaces $\left(F_{p}\right)_{p \in N}$ of $H$ satisfying

$$
\mu\left(F_{p} \otimes F_{q}\right) \subset F_{p+q} \Delta\left(F_{p}\right) \subset \sum_{0 \leqslant q \leqslant p} F_{q} \otimes F_{p-q} \underset{p \in N}{\cup} F_{p}=H .
$$

With respect to the graded cocommutative Hopf algebra $\Omega$, we remark that $F_{p}=\oplus_{0 \leqslant q \leqslant p} \Omega^{q}$ defines a compatible filtering. Due to the graded cocommutativity, $P(\Omega)$ does not have the struc- 
ture of an ordinary Lie algebra. Instead of the usual commutator we define a "color" commutator on $\Omega$ by $[x, y]=x y-(-1)^{p q} y x$ for $x \in \Omega^{p}$ and $y \in \Omega^{q}$. The set $P(\Omega)$ is closed under this bracket since

$$
\begin{aligned}
\Delta([x, y]) & =\Delta\left(x y-(-1)^{p q} y x\right) \\
& =\Delta(x) \Delta(y)-(-1)^{p q} \Delta(y) \Delta(x) \\
& =(1 \otimes x+x \otimes 1)(1 \otimes y+y \otimes 1)-(-1)^{p q}(1 \otimes y+y \otimes 1)(1 \otimes x+x \otimes 1) \\
& =1 \otimes x y+x \otimes y+(-1)^{p q} y \otimes x+x y \otimes 1-(-1)^{p q}\left(1 \otimes y x+y \otimes x+(-1)^{p q} x \otimes y+y x \otimes 1\right) \\
& =1 \otimes\left(x y-(-1)^{p q} y x\right)+\left(x y-(-1)^{p q} y x\right) \otimes 1 \\
& =1 \otimes[x, y]+[x, y] \otimes 1,
\end{aligned}
$$

for all $x \in P(\Omega)^{p}$ and $y \in P(\Omega)^{q}$. Note that the $\mathbb{N}$ grading of $\Omega$ induces an $\mathbb{N}$ grading on $P(\Omega)$, since $\Delta$ is homogeneous of degree zero. By this argument we have derived that $P(\Omega)$ is a color Lie superalgebra. For the definition of a color Lie superalgebra we refer to Ref. 12. The corresponding 2-cocyle $\alpha$ is given by

$$
\alpha: \mathbb{N} \times \mathbb{N} \rightarrow \mathrm{C}^{*}, \quad \alpha(p, q)=(-1)^{p q} .
$$

Since $\alpha(q, q)=(-1)^{q^{2}}=(-1)^{q}$, the elements of $P(\Omega)^{q}$ are even (odd) if and only if $q$ is even (odd). As in the case of ordinary Lie algebras, one can define the universal enveloping algebra of a color Lie superalgebra and a corresponding Hopf algebraic structure on it. Analogous to the above mentioned result of Ref. 11, the Hopf algebra $\Omega$ is isomorphic to the Hopf algebra $U(P(\Omega))$.

The question is what $P(\Omega)$ explicitly looks like. The set of primitive elements of $\Omega$ certainly contains the linear span of $\left\{x^{1}, x^{2}, \ldots, x^{n}, y^{1}, y^{2}, \ldots, y^{n}\right\}$ [see (17)], which we will denote by $L$. We consider $L$ as the $\mathbb{N}$-graded vector space $L=\oplus_{p \in N} L^{p}$ with

$$
L^{0}=\left\langle x^{i}\right\rangle_{1 \leqslant i \leqslant n}, \quad L^{1}=\left\langle y^{i}\right\rangle_{1 \leqslant i \leqslant n} \quad L^{p}=0 \quad(p \geqslant 2) .
$$

According to the relations (2) and (21), we have

$$
\left[x^{i}, x^{j}\right]=C_{k}^{i j} x^{k}, \quad\left[x^{i}, y^{j}\right]=A_{k}^{i j} y^{k}, \quad \text { and } \quad\left[y^{i}, y^{j}\right]=0 \text {, }
$$

so $L$ is a color Lie supersubalgebra in $P(\Omega)$. Since the elements of $L^{0}$ are even and the elements of $L^{1}$ are odd, a basis of $U(L)$ can be given by (see, e.g., Ref. 12)

$$
y^{j_{1}} y^{j_{2} \cdots y^{j_{q}} x^{i_{1}} x^{i_{2}} \cdots x^{i_{p}}} \text { with } j_{1}<j_{2}<\cdots<j_{q}, \quad i_{1} \leqslant i_{2} \leqslant \cdots \leqslant i_{p}, \quad \text { and } p, q \geqslant 0 .
$$

A comparison of this basis with the described basis of $\Omega$ [see (3)], proves that the Hopf algebra $\Omega$ is isomorphic to the Hopf algebra $U(L)$. Hence, $P(\Omega)=L$.

The preceding reasoning enables us to interpret the construction of a differential Hopf algebra on the universal enveloping of a Lie algebra $\mathfrak{g}$ in terms of a color Lie superalgebraic extension of g. The problem to be solved can be reformulated as follows. Given is a Lie algebra $\mathfrak{g}$ with basis $\left\{x^{1}, x^{2}, \ldots, x^{n}\right\}$. Let $L$ be the $\mathrm{N}$-graded vector space given by $L^{0}=\mathfrak{g}, L^{1}=\left\langle y^{i}\right\rangle_{1 \leqslant i \leqslant n}$ and $L^{p}=0$ for all $p \geqslant 2$. Define a bilinear operation [, ] on $L$ of degree zero extending the commutator of $\mathfrak{g}$ in such a way that $\left[x^{i}, y^{j}\right]=A_{k}^{i j} y^{k}$. This bracket expresses the commutation relations (21). The conditions (20) for the coefficients $A_{k}^{i j}$ are equivalent to demanding that $L$ equipped with this commutator becomes a color Lie superalgebra with 2-cocycle $\alpha$ given by (26), satisfying the additional property that the linear map $d$ defined by $d\left(x^{i}\right)=y^{i}$ and $d\left(y^{i}\right)=0$ is a graded derivation of degree 
1 on $L$. The corresponding differential Hopf algebra on $U(\mathfrak{g})$ is then given by the Hopf algebra $U(L)$ equipped with the unique extension of the derivation $d$ from $L$ to $U(L)$.

\section{CONCLUDING REMARKS}

We have presented a framework to construct a De Rham complex on the universal enveloping algebra of a Lie algebra $\mathfrak{g}$. The fundamental property of the differential algebra is that it possesses a so-called PBW basis. We have proven that the differential algebra can be given a Hopf algebra structure extending the natural Hopf algebra $U(\mathfrak{g})$. In our presentation we assumed $\mathfrak{g}$ to be finite dimensional. This is definitely not a necessary condition; one can easily see that this framework can also be applied in the infinite-dimensional case. For more details on this and some explicit examples we refer to Ref. 13. Naturally, a De Rham complex on $U(\mathfrak{g})$ brings to surface the notion of cohomology. It would be very interesting to investigate this De Rham cohomology.

'S. L. Woronowicz, "Differential calculus on compact matrix pseudogroups (quantum groups)," Commun. Math. Phys. 122, 125 (1989).

${ }^{2}$ A. Connes, "Non-commutative differential geometry," Publ. Math. I.H.E.S. 62, 257 (1986).

${ }^{3}$ J. Wess and B. Zumino, "Covariant differential calculus on the quantum hyperplane," Nucl. Phys. 188, 303-312 (1990).

${ }^{4} \mathrm{G}$. Maltsiniotis, "Groupes quantiques et structures differentielles (quantum groups and differential structures)," C.R. Acad. Sci. Paris, Sér I. 311, 831-834 (1990).

${ }^{5}$ M. Dubois-Violette, R. Kerner, and J. Madore, "Noncommutative differential geometry of matrix algebras," J. Math. Phys. 31, 316-322 (1990).

${ }^{6}$ Yu. I. Manin, "Notes on quantum groups and quantum De Rham complexes," preprint MPI/91-60, Bonn, 1991.

${ }^{7}$ H. C. Baehr, A. Dimakis, and F. Müller-Hoissen, "Differential calculi on commutative algebras," preprint, Max-PlanckInstitut für Physik, Munich, 1994.

${ }^{8}$ V. G. Drinfel'd, Quantum Groups, Proceedings of the International Congress of Mathematicians, Berkeley, 1986, edited by A. M. Gleason (American Mathematical Society, Providence, RI, 1987), pp. 798-820.

${ }^{9}$ G. M. Bergman, "The diamond lemma for ring theory," Adv. Math. 29, 178-218 (1978).

${ }^{10}$ E. Abe, Hopf Algebras (Cambridge University, Cambridge, 1980).

${ }^{11} \mathrm{~N}$. Bourbaki, "Elements de mathématique, groupes et algèbres de Lie," Diffusion CCLS, 1972, Chaps. 2-3.

${ }^{12}$ Yu. A. Bahturin, A. A. Mikhalev, V. M. Petrogradsky, and M. V. Zaicev, Infinite Dimensional Lie Superalgebras (Walter de Gruyter, Berlin, 1992).

${ }^{13}$ R. Martini, G. F. Post, and P. H. M. Kersten, "Differential calculus on universal enveloping algebras of Lie algebras," Memorandum 1261, University of Twente, Enschede, 1995. 